\title{
Comparative investigation on radical polymerization of methyl and ethyl methacrylate under multi-site phase-transfer catalytic conditions
}

\author{
Vajjiravel Murugesan $^{1} \cdot$ Elumalai Marimuthu ${ }^{1}$
}

Received: 6 October 2017 / Accepted: 4 January 2018 / Published online: 25 January 2018

(c) The Author(s) 2018. This article is an open access publication

\begin{abstract}
Methyl and ethyl methacrylate was polymerized in heterogeneous system with the help of newly synthesized multi-site phase-transfer catalyst and using water-soluble initiator at $60 \pm 1{ }^{\circ} \mathrm{C}$ under unstirred inert atmospheric condition. Polymer yield was increased with increasing molar concentrations of monomer, initiator, catalyst and temperature. Polymerization follows first-order kinetics with respect to monomer and half-order with respect to catalyst and initiator, respectively. PTC has myriads of applications in the synthesis of various organic and polymeric materials because of its fast reaction and high yield in short period of time. Without addition of PTC, polymerization did not occur; this indicates that catalyst plays the pivotal role on initiation of polymerization. It extracts the reactive radical anion from aqueous phase and transfers to the organic phase where acrylates were polymerized. Polymerization reactivity of methyl and ethyl methacrylate under PTC conditions was studied by various parameters. The activation energy (Ea) and other thermodynamic parameters were calculated. The Ea value supports the reactivity of acrylates. The results obtained from this investigation were used for inferring the radical mechanism of phase-transfer-catalyzed polymerization. The obtained polymers were analyzed by spectral and thermal analyses.
\end{abstract}

Keywords Kinetics $\cdot$ Multi-site phase-transfer catalyst $\cdot$ Rate of polymerization · Two-phase system $\cdot$ Methyl and ethyl methacrylate

\section{Introduction}

Acrylates containing polymers have potential applications in industries like textile, surface coating, automotive, aerospace, biomedical to optics and microelectronics, etc. because of their surface-free energy properties and water/ oil repellence, etc. [1, 2]. Synthesis of acrylates polymers with controlled molecular weight distributions and low polydispersity index (PDI) is one of the key parameters to realize any kind of applications and synthesis of such acrylates polymers has attracted remarkable attention between polymer chemists and industrial community. Among the various polymerization techniques, free radical polymerization is one of the simplest and well-known best techniques for

Vajjiravel Murugesan

chemvel@rediffmail.com; vajjiravel_m@bsauniv.ac.in

1 Department of Chemistry, B S Abdur Rahman Crescent Institute of Science and Technology, Chennai 600 048, India synthesizing poly (alkyl acrylates/alkyl methacrylates) with different composition, structures, and functionalities and with low dispersity, as this polymerization can be carried out at room and or moderately high temperature with lessstringent reaction condition compared to anionic polymerization [3-9].

The discovery and establishment of nitroxide-mediated stable free radical polymerization (NMP) [3, 7], radical addition fragmentation chain transfer polymerization (RAFT) $[5,8]$, atom transfer radical polymerization (ATRP) $[4,6]$ and degenerative chain transfer polymerization $[10,11]$ contributed an incredible advancement in the area of controlled living radical polymerization of alkyl acrylates/alkyl methacrylates for the past few decades. Consequently, in the verge of these polymerization techniques, phase-transfer-catalyzed polymerization is one of the recognized and established techniques for the polymerization of alkyl methacrylates in heterogeneous condition using water-soluble initiator. PTC is the most important innovative technique for promoting reactions between lipophilic and hydrophilic reactants. In 
which one reactant is lipophilic and the other is hydrophilic, high rates of reaction can be attained by adding PTC and it helps to overcome the immiscibility of the reactants during the reaction. In this technique, the monomer (acrylates) was dissolved in the organic solvent and initiator (PDS) is dissolved in water, these mixtures are immiscible in nature and then by adding PTC, polymerization reaction occur through the transport of one reactant into the other phase. The advantages of PTC are well documented in the literature [12-15].

There are several reports on polymerization of various alkyl methacrylates was reported using different PTC [16-27]. According to these reports, the comparative and reactivity of methyl and ethyl methacrylate under multi-site phase-transfer catalytic conditions is limited. In this context, herein we report the polymerization of methyl and ethyl methacrylate under multi-site phase-transfer catalytic conditions. In this study, we observe that the reactivity of EMA is faster than MMA on the rate of polymerization and it was supported by Arrhenius plot. Further, the influence of various reaction parameters on the rate of polymerization $(R \mathrm{p})$ of methyl and ethyl methacrylate was studied and discussed in detail.

\section{Experimental}

\section{Reagents and solvents}

Methyl methacrylate (MMA) and ethyl methacrylate (EMA) supplied by Lancaster, India were purified by washing with 5 $\mathrm{wt} \%$ of sodium hydroxide to remove inhibitor and then dried with calcium chloride overnight and distilled over calcium hydride under reduced pressure prior to use. The initiator potassium peroxydisulphate (PDS, Aldrich, India) was purified twice by recrystallization in cold water. The multi-site phase-transfer catalyst (TMBPEDBC as multi-site PTC) was synthesized by adopting reported procedure [24, 25]. All the solvents were of reagent grade and were purchased from Finar, Avra, India. The double distilled water is used to make an aqueous phase.

\section{Synthesis of multi-site phase-transfer catalyst (TMBPEDBC)}

$N, N, N^{\prime}, N^{\prime}$-tetramethylethylenediamine $(0.01 \mathrm{~mol})$ was dissolved in ethanol $(10 \mathrm{~mL})$, taken in a two-necked roundbottom flask and stirred at $70{ }^{\circ} \mathrm{C}$. The $n$-propyl bromide (0.01 mol dissolved in ethanol) was added dropwise. After $1 \mathrm{~h}$, a solution of benzyl chloride $(0.01 \mathrm{~mol}$ dissolved in ethanol) was added slowly to the reaction mixture. The reaction mixture was gently refluxed and stirred for $24 \mathrm{~h}$. After that, the mixture was cooled to room temperature and the solvent was evaporated. The white precipitate of $1,1,2$, 2-tetramethyl-1-benzyl-2-n-propylethylene-1, 2-diammonium bromide chloride (TMBPEDBC) was obtained. It was purified by dissolving a minimum amount of catalyst in ethanol and diffusing over hexane; pure white precipitate was collected and dried $[24,25]$. The yield and melting point of the catalyst were $78 \%$ and $210{ }^{\circ} \mathrm{C}$, respectively (Scheme 1).

\section{Polymerization of methyl and ethyl methacrylate}

Polymerization experiments were carried out in a specially designed borosil glass tube with dimensions of 30 and $26 \mathrm{~mm}$ for outer and inner diameter, respectively, and $120 \mathrm{~mm}$ height. The polymerization glass tube consists of equal volume of organic and aqueous phase. The monomer in ethyl acetate was the organic phase and aqueous phase containing specified concentrations of initiator, catalyst and acid (for maintaining $\mathrm{pH}$ ). The tube containing above mixture was bubbled by passing nitrogen gas for $20 \mathrm{~min}$ to remove dissolved oxygen completely from polymerization system and then it was sealed. The reaction tube was then placed in a temperature-controlled water bath at $60 \pm 1{ }^{\circ} \mathrm{C}$ (Fig. 1). After the stipulated time, polymerization was terminated by pouring reaction mixture into cold methanol. Finally, the resultant polymer was filtered (Fig. 2) and dried in vacuum oven at $60{ }^{\circ} \mathrm{C}$.

The rate of polymerization $(R \mathrm{p})$ was calculated from the gravimetric determination of the polymer formed in a given time of polymerization. The $R \mathrm{p}$ was calculated from the weight of polymer obtained using the formula: $R \mathrm{p}=1000 \mathrm{~W} / V \times t \times M$, where $W$ is the weight of the polymer in gram, $V$ is the volume of the reaction mixture in $\mathrm{ml} ; t$ is the reaction time in seconds; and $M$ is the molecular weight of the monomer in $\mathrm{g} / \mathrm{mol}$. The kinetic experiments were carried out by changing the concentration of monomer, initiator, catalyst and temperature, etc. by adopting aforementioned polymerization procedure as depicted in Scheme 2.

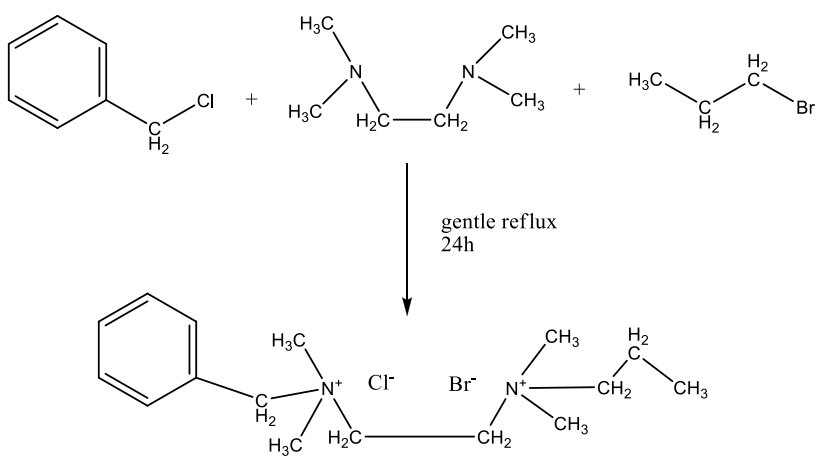

Scheme 1 Synthesis of multi-site PTC: 1, 1, 2, 2-tetramethyl-1-benzyl-2-n-propylethylene-1, 2-diammonium bromide chloride (TMBPEDBC) 


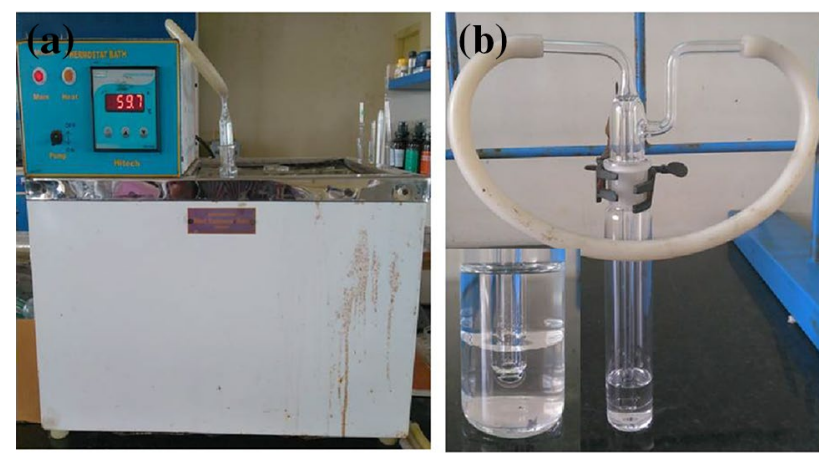

Fig. 1 Polymerization reaction setup: a Temperature-controlled water bath, b Polymerization reaction tube consists of equal volume of aqueous and organic phase
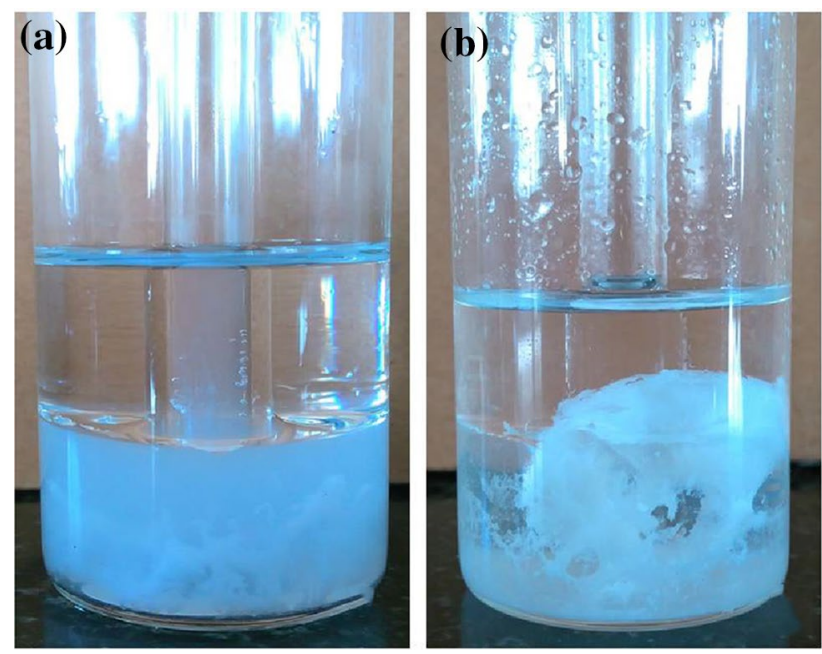

Fig. 2 Differences in the rate of precipitations of a poly (methyl methacrylate) and b poly (ethyl methacrylate)
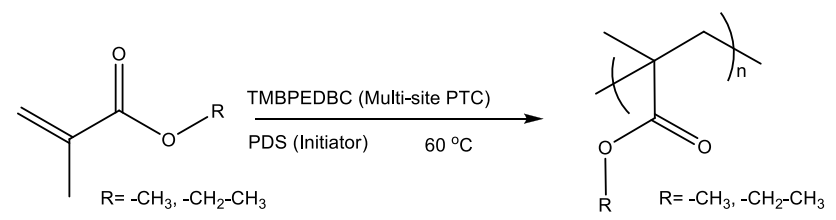

Scheme 2 Multi-site phase transfer catalyzed radical polymerization of methyl and ethyl methacrylate in two-phase system

\section{Instruments}

The FT-IR spectrum of poly (alkyl methacrylate) was recorded on an FT-IR spectrometer (JASCO) in the spectral region from 3500 to $500 \mathrm{~cm}^{-1}$. A pellet of polymer samples was made with $\mathrm{KBr}$ on recording of spectrum. The thermal analyses of poly(methyl methacrylate) and poly (ethyl methacrylate) were carried out using TA (DSC Q10, TGA Q600) instruments; Pyris diamond DSC/TGA analyzer; sample weight $3.0540 \mathrm{mg}$ of PMMA and $4.9055 \mathrm{mg}$ of PEMA at heating rate $15^{\circ} \mathrm{C}$ per minutes with the temperature range of $30-600{ }^{\circ} \mathrm{C}$. The wide-angle X-ray diffraction was recorded using PAN Analytical Xpert PRO instrument operated at the $\mathrm{Cu} \mathrm{K} \alpha$ radiation wavelength of $1.54 \AA$ at $45 \mathrm{~mA}$ and $40 \mathrm{kV}$.

\section{Reaction model}

In heterogeneous reactions, the rate of reaction depends on the interaction and diffusion between two phases. The reaction between two immiscible reactants is low due to less collision between them. The common way to address such difficulty is to carry out the reaction at high temperature and using a co-solvent. However, such conditions are generally limited because of side reactions and complexity in choosing co-solvent. Generally, in a heterogeneous medium the rate of reaction dramatically enhanced with the help of phasetransfer catalyst (PTC). PTC is capable of extracting and transferring ionic reactants of aqueous and or solid phase into organic phase, where the reaction will take place. An extraction mechanism was presumed for the polymerization process, the quaternary ammonium salt $(\mathrm{QX})$ is dissolve well in the aqueous phase and react with initiator (KY), then produces radical anion $(\mathrm{QY})$ at the interface between the phases. Subsequently, PTC transfers the radical anion (QY) from the aqueous phase into organic phase where the polymerization occurs at $60 \pm 1{ }^{\circ} \mathrm{C}$. A typical phase-transfer-catalyzed radical polymerization cyclic process is shown in Scheme 3.

\section{Results and discussion}

In an attempt to verify the reaction kinetics model and explore the role of monomers, initiator, catalyst, temperature and aqueous phase variation on the rate of polymerization ( $\mathrm{Rp}$ ) were investigated in cyclohexane-water two-phase systems at $60 \pm 1{ }^{\circ} \mathrm{C}$.

\section{Steady-state rate of polymerization}

The plot of $R \mathrm{p}$ versus time for the polymerization of methyl and ethyl methacrylate (RMA) using initiator (PDS), multisite PTC (TMBPEDBC) and with the fixed concentrations of all other parameters is shown in Fig. 3. The rate of polymerization $\left(R_{\mathrm{p}}\right)$ increases nicely to some extent, slightly decreases thereafter and reaches the constant value [22]. The steady-state rate of polymerization of RMA was arrived at $50 \mathrm{~min}$. The role of various reaction parameters on the rate of polymerization was studied at steady-state time. 


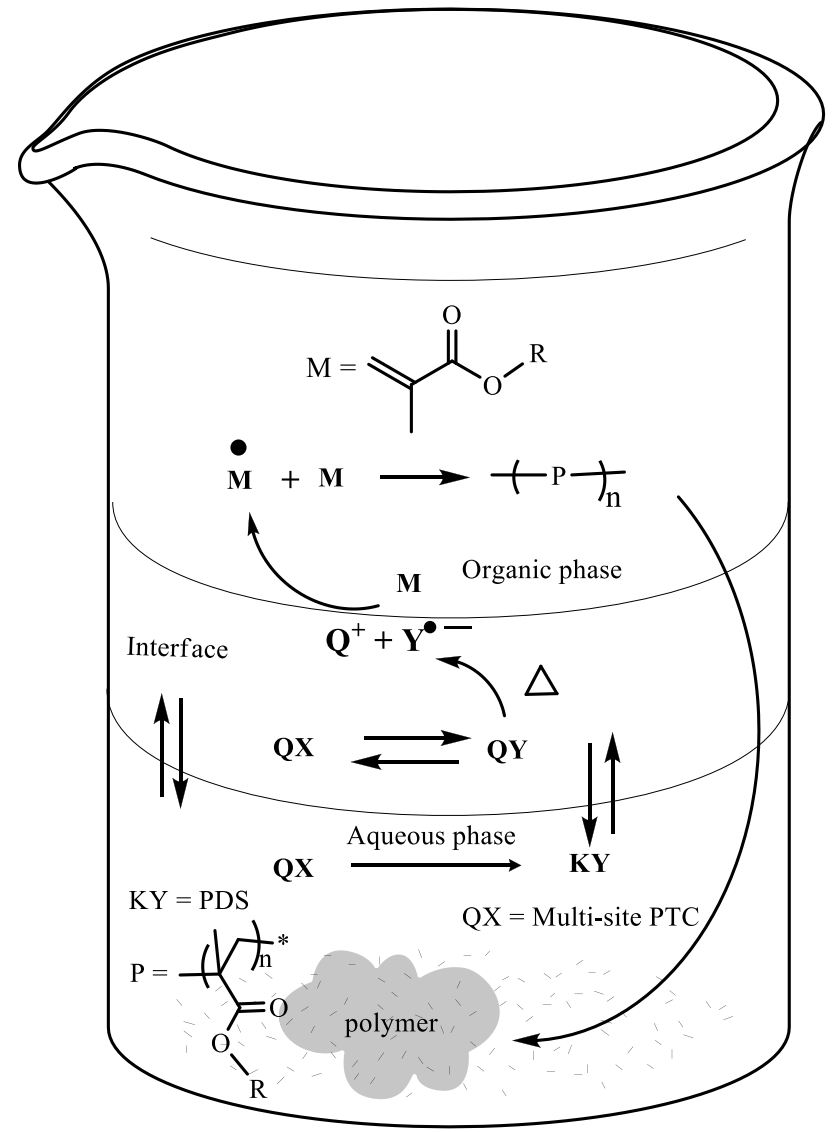

Scheme 3 Reaction model for the polymerization of alkyl methacrylate in two-phase system

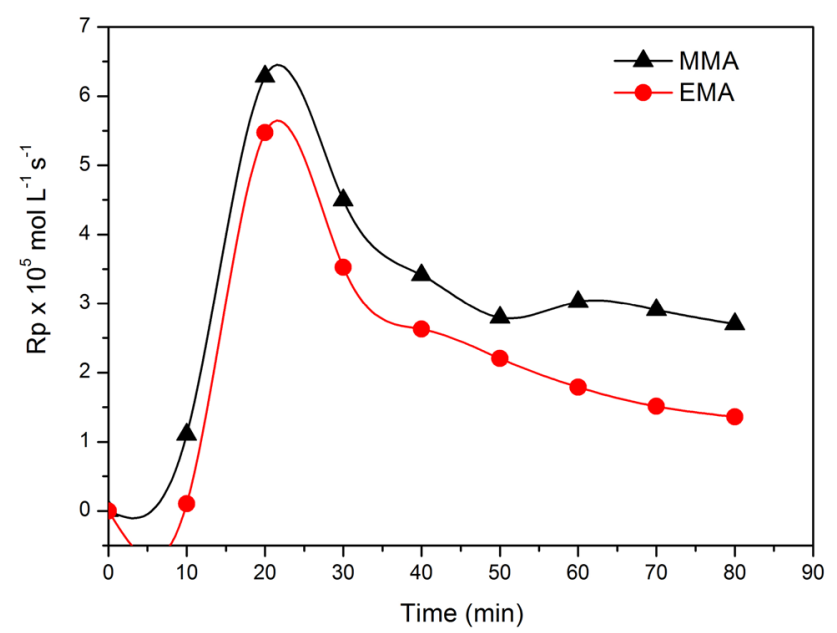

Fig. 3 Steady-state rate of polymerization. Reaction condition: [RMA]: $2.0 \mathrm{~mol} \mathrm{dm}{ }^{-3}$; [PDS]: $2.0 \times 10^{-2} \mathrm{~mol} \mathrm{dm}^{-3}$; [TMBPEDBC]: $\left.2.0 \times 10^{-2} \mathrm{~mol} \mathrm{dm}^{-3} ; \mathrm{H}^{+}\right]: 0.50 \mathrm{~mol} \mathrm{dm}^{-3} ;[\mu]: 0.20 \mathrm{~mol} \mathrm{dm}^{-3}$; Temperature: $60 \pm 1^{\circ} \mathrm{C}$

\section{Role of [RMA] on Rp}

Generally, most of the polymerization rate is first-order dependence with respect to monomer concentration. We have studied polymerization rate of the methyl and ethyl methacrylate concentrations in the range of $4.5-9.5 \mathrm{~mol} \mathrm{dm}^{-3}$ by keeping other parameters constant. $R_{\mathrm{p}}$ increases with an increase in the concentration of the monomers. We observed that the polymerization rate is higher for EMA as compared to MMA is may attributed to possession electron donating group in EMA. Figure 4 shows the half-order relationship for the polymerization of methyl and ethyl methacrylate. The plot of $R_{\mathrm{p}}$ versus [RMA] passing through the origin confirms the above observations with respect to [RMA]. The deviation from the first-order relationship is due to the dependence of initiation rate on the monomer concentration. The half-order with respect to concentration of monomer
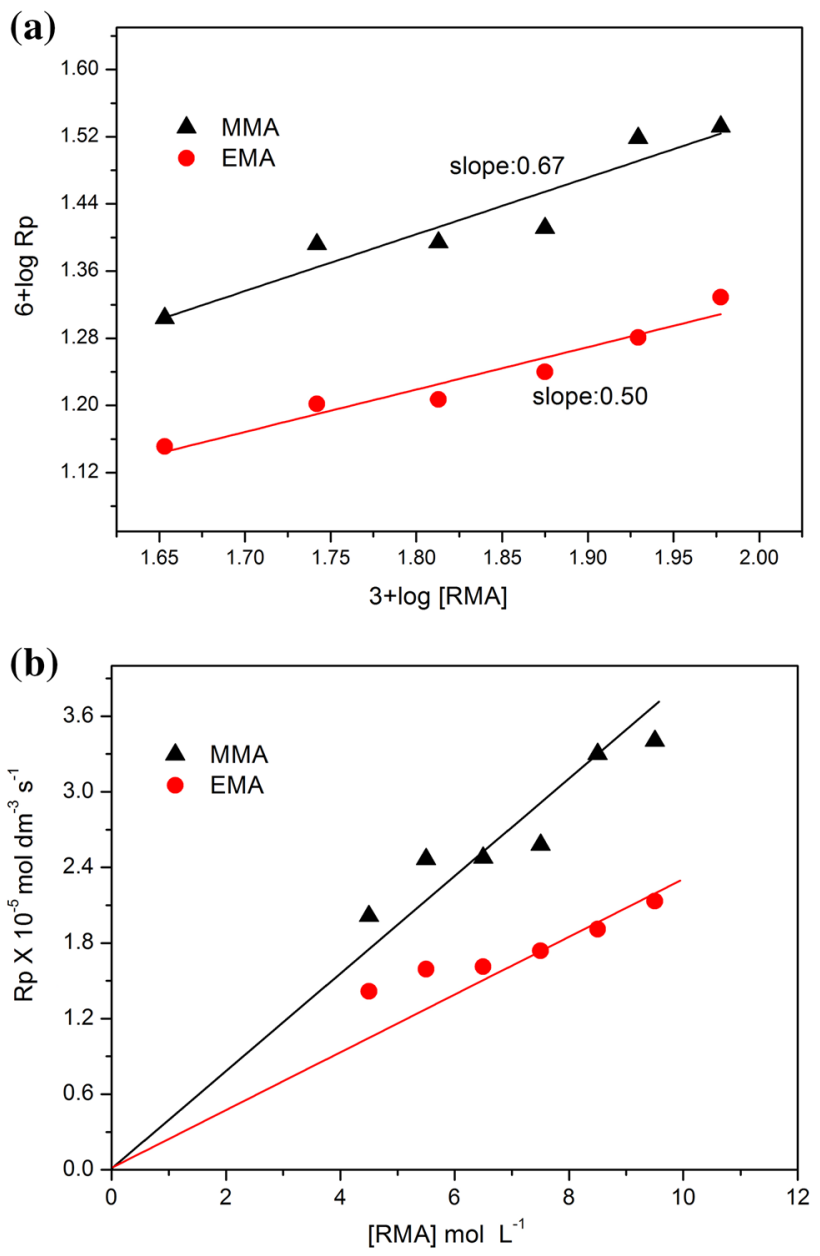

Fig. 4 Role of [RMA] on the $R$ p. a Plot of $6+\log$ Rp versus $3+\log$ [RMA]. b Plot of Rp versus [RMA]. Reaction condition: [PDS]: $2.0 \times 10^{-2} \mathrm{~mol} \mathrm{dm}^{-3}$; [TMBPEDBC]: $2.0 \times 10^{-2} \mathrm{~mol} \mathrm{dm}^{-3} ;\left[\mathrm{H}^{+}\right]$: $0.50 \mathrm{~mol} \mathrm{dm}^{-3} ;[\mu]: 0.20 \mathrm{~mol} \mathrm{dm}^{-3}$; Temperature: $60 \pm 1{ }^{\circ} \mathrm{C}$; Time: $50 \mathrm{~min}$ 
has been reported for the polymerization of n-butyl methacrylate and ethyl methacrylate with other multi-site PTC using potassium peroxydisulphate as initiator [24, 26, 27].

\section{Role of [PDS] on $\boldsymbol{R}_{\mathrm{p}}$}

To check the role of initiator on polymerization rate, we conducted the polymerization using the initiator concentrations in the range of $1.5-2.5 \mathrm{~mol} \mathrm{dm}^{-3}$ at fixed concentrations of other parameters. It was observed that the rate of polymerization increases with rise of initiator concentration. Figure 5a shows initiator dependence order value of 0.59 and 0.80 for MMA and EMA, respectively. It was obtained from the plot of $6+\log R_{\mathrm{p}}$ versus $3+\log$ [PDS]. As expected, the plot of $R_{\mathrm{p}}$ versus [PDS] is linear passing through the origin supporting the above deduction in Fig. 5b. Generally, polymerization rate is to be dependent on the square root of the initiator concentration. The deviation of square root relationship of initiator is due to the termination occurring by combination of primary radicals and gel effect or diffusion-controlled termination constant [16].

\section{Role of [TMBPEDBC] on $\boldsymbol{R}_{\mathrm{p}}$}

Polymerization rate was explored using multi-site PTC (TMBPEDBC) in the concentration range of $1.5-2.5 \mathrm{~mol} \mathrm{dm}^{-3}$ at fixed other parameters. It was found that the rate of polymerization of MMA and EMA increases with increasing concentration of catalyst. The amount and formation of $\mathrm{QS}_{2} \mathrm{O}_{8}$ (quaternary ammoniumperoxydisulphate complex) are increased with increasing catalyst (dual active-site) concentration. The highly concentrated $\mathrm{QS}_{2} \mathrm{O}_{8}$ complex is decomposed and produces more radical anion which accelerates the polymerization fast and it may lead to increase in the polymerization rate. As shown in Fig. 6a, the plot of $6+\log R_{\mathrm{p}}$ versus $3+\log$ [TMBPEDBC], the order with respect to catalyst was found to be 0.63 and 0.42 for MMA and EMA. The observed order was confirmed by the plot of $R_{\mathrm{p}}$ versus [TMBPEDBC] as shown in Fig. 6b. To establish the role of catalyst in this polymerization system, we performed the blank experiment: First of all, we observed that the PDS alone did not initiate the polymerization in twophase system under our reaction conditions. The changes in the appearance of two-phase media was observed (slight turbid) but while pouring the reaction mixture into methanol it was disappeared. The polymerization did not occur in the absence of catalyst even after several minutes. This observation was the proof for role of catalyst on the polymerization reaction.
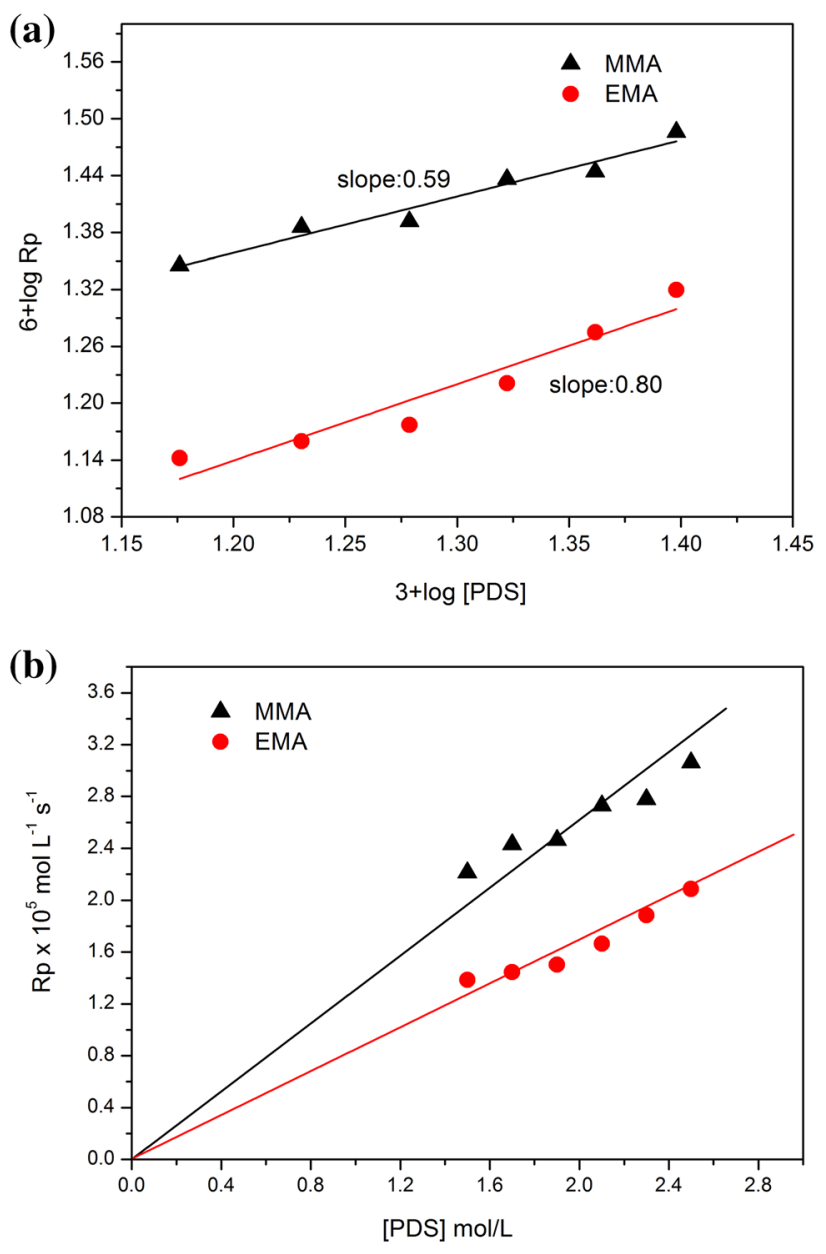

Fig. 5 Role of [PDS] on the Rp. a Plot of $6+\log \mathrm{Rp}$ versus $3+\log$ [PDS]. b Plot of Rp versus [PDS]. Reaction condition: [RMA]: $2.0 \mathrm{~mol} \mathrm{dm}^{-3}$; [TMBPEDBC]: $2.0 \times 10^{-2} \mathrm{~mol} \mathrm{dm}^{-3} ;\left[\mathrm{H}^{+}\right]$: $0.50 \mathrm{~mol} \mathrm{dm}{ }^{-3}[\mu]: 0.20 \mathrm{~mol} \mathrm{dm}^{-3}$; Temperature: $60 \pm 1 \mathrm{C}$; Time: $50 \mathrm{~min}$

\section{Role of temperature on $R_{\mathrm{p}}$}

The role of temperature $(T)$ on the polymerization reaction was varied from 50 to $65^{\circ} \mathrm{C}$. The plot of $\log R \mathrm{p}$ versus $1 / T$ is shown in Fig. 7. The gradual increase in Rp with varying temperature was observed. An increase in the rate of polymerization is attributed to fast formation rate of radicals and intensive nucleation which are responsible for accelerating the polymerization process promptly and, thereby, the rate of polymerization was increased significantly. The Arrhenius equation with activation energy of polymerization (Ea) was found to be 10.20 and $8.59 \mathrm{k} \mathrm{J} / \mathrm{mol}$ for MMA and EMA, respectively. The lower value of Ea of EMA suggested that the polymerization reaction occurs quickly as compared with MMA under multi-site phase-transfer catalytic condition. The thermodynamic parameters such as entropy of activation $\left(\Delta S^{\#}\right)$, enthalpy of activation $\left(\Delta H^{\#}\right)$ and free energy

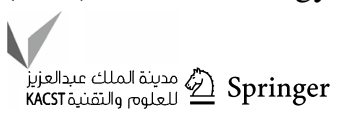




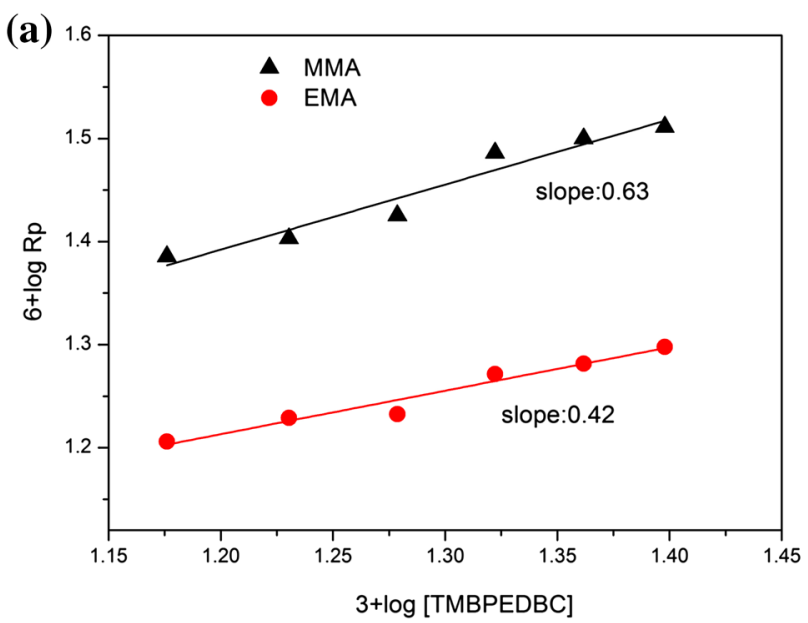

(b)

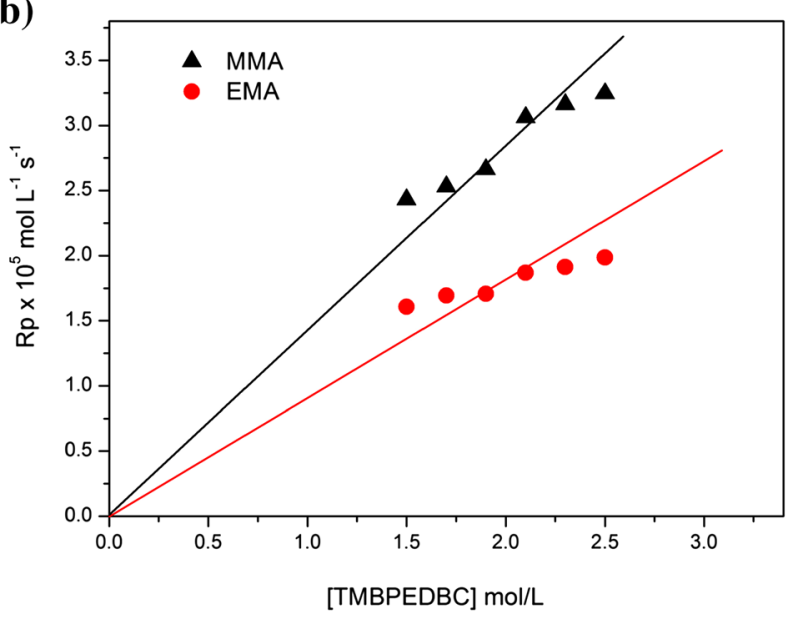

Fig. 6 Role of [TMBPEDBC] on the $R$ p. a Plot of $6+\log$ Rp versus $3+\log$ [TMBPEDBC]. b Plot of Rp versus [TMBPEDBC]. Reaction condition: [RMA]: $2.0 \mathrm{~mol} \mathrm{dm}^{-3}$; $\left.\mathrm{PDS}\right]: 2.0 \times 10^{-2} \mathrm{~mol} \mathrm{dm}^{-3}$; $\left[\mathrm{H}^{+}\right]$: $0.50 \mathrm{~mol} \mathrm{dm}^{-3 ;}[\mu]: 0.20 \mathrm{~mol} \mathrm{dm}^{-3}$; Temperature: $60 \pm 1{ }^{\circ} \mathrm{C}$; Time: $50 \mathrm{~min}$

of activation $\left(\Delta G^{\#}\right)$ have been calculated and presented in Table 1. The comparison of Ea value for different alkyl methacrylate under phase-transfer catalytic polymerization was reported [20, 28, 29]. The overall activation energy of $72.80 \mathrm{~kJ} / \mathrm{mol}$ was reported for the polymerization of MMA using diphenyldisulphide and $\mathrm{Cu}$ (II) ion system [33].

\section{Role of organic solvents polarity on $R_{\mathrm{p}}$}

The role of organic solvents polarity on the $R_{\mathrm{p}}$ was examined under aforementioned conditions. The organic solvents involved in the investigation are cyclohexane, ethylacetate and cyclohexanone having the dielectric constants 2.02, 6.02 and 18.03 , respectively. It was found that the $R_{\mathrm{p}}$ decreased in the following order: cyclohexanone $>$ ethyl acetate $>$ cyclohexane. An increase in the rate of polymerization was attributed to the increase in the polarity of the solvents,

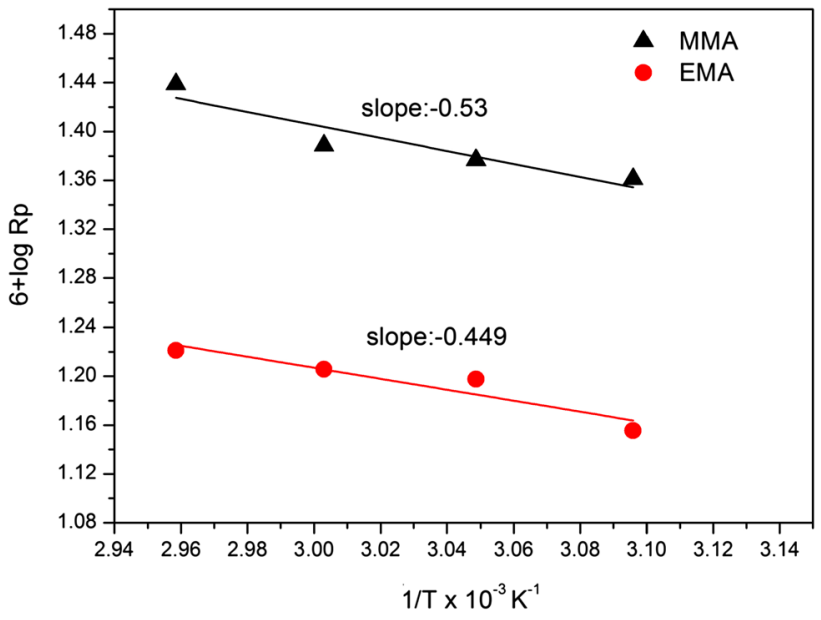

Fig. 7 Role of temperature on the $R \mathrm{p}$ Reaction condition: [RMA]: $2.0 \mathrm{~mol} \mathrm{dm}{ }^{-3}$ [PDS]: $2.0 \times 10^{-2} \mathrm{~mol} \mathrm{dm}^{-3}$; [TMBPEDBC]: $2.0 \times 10^{-2} \mathrm{~mol} \mathrm{dm}^{-3}\left[\mathrm{H}^{+}\right]: 0.50 \mathrm{~mol} \mathrm{dm}{ }^{-3}[\mu]: 0.20 \mathrm{~mol} \mathrm{dm}^{-3}$; Time: $50 \mathrm{~min}$

Table 1 Thermodynamic parameters of RMA

\begin{tabular}{lclll}
\hline Monomer & Ea kJ/mol & $\Delta G^{\#} \mathrm{~kJ} / \mathrm{mol}$ & $\Delta H^{\#} \mathrm{~kJ} / \mathrm{mol}$ & $\Delta S^{\#} \mathrm{~kJ} / \mathrm{mol}$ \\
\hline MMA & 10.20 & 40.96 & 4.118 & -110.68 \\
EMA & 8.59 & 43.89 & 3.28 & -121.98 \\
\hline
\end{tabular}

Table 2 Role of organic solvents polarity on Rp

\begin{tabular}{lcll}
\hline Solvents & $\begin{array}{l}\text { Dielectric con- } \\
\text { stant }(\varepsilon)\end{array}$ & $\begin{array}{l}R \mathrm{p} \times 10^{5} \mathrm{~mol} \\
\mathrm{~L}^{-1} \mathrm{~s}^{-1} \\
(\mathrm{MMA})\end{array}$ & $\begin{array}{l}R \mathrm{p} \times 10^{5} \\
\mathrm{~mol} \mathrm{~L}^{-1} \\
\mathrm{~s}^{-1} \\
(\mathrm{EMA})\end{array}$ \\
\hline Cyclohexanone & 18.3 & 3.04 & 2.83 \\
Ethyl acetate & 3.91 & 2.90 & 1.96 \\
Cyclohexane & 2.02 & 2.02 & 1.09 \\
\hline
\end{tabular}

[RMA]: $2.0 \mathrm{~mol} \mathrm{~L} \mathrm{~L}^{-1 ;}[\mathrm{PDS}]: 2.0 \times 10^{-2} \mathrm{~mol} \mathrm{~L}^{-1}$; [TMBPEDBC]: $2.0 \times 10^{-2} \mathrm{~mol} \mathrm{~L}^{-1} ;\left[\mathrm{H}^{+}\right]: 0.5 \mathrm{~mol} \mathrm{~L}^{-1} ;[\mu]: 0.2 \mathrm{~mol}$ $\mathrm{L}^{-1}$; Temperature: $60 \pm 1^{\circ} \mathrm{C}$; Time: $50 \mathrm{~min}$

which facilitates greater transfer of peroxydisulfate ion from aqueous phase to organic phase [27-29] (Table 2).

\section{Role of variation of aqueous phase on $R_{p}$}

The role of aqueous (liquid) phase is playing the pivotal role in the phase-transfer-catalyzed reactions. The role of aqueous phase on the rate of polymerization of MMA and EMA under phase-transfer catalytic conditions was explored in polymerization and the reactions were conducted with a constant volume of organic phase and changing the volumes of aqueous phase $\left(V_{\mathrm{w}} / V_{\mathrm{o}}=0.29-0.90\right)$ at fixed concentrations of all other parameters for MMA and EMA (Fig. 8). The 


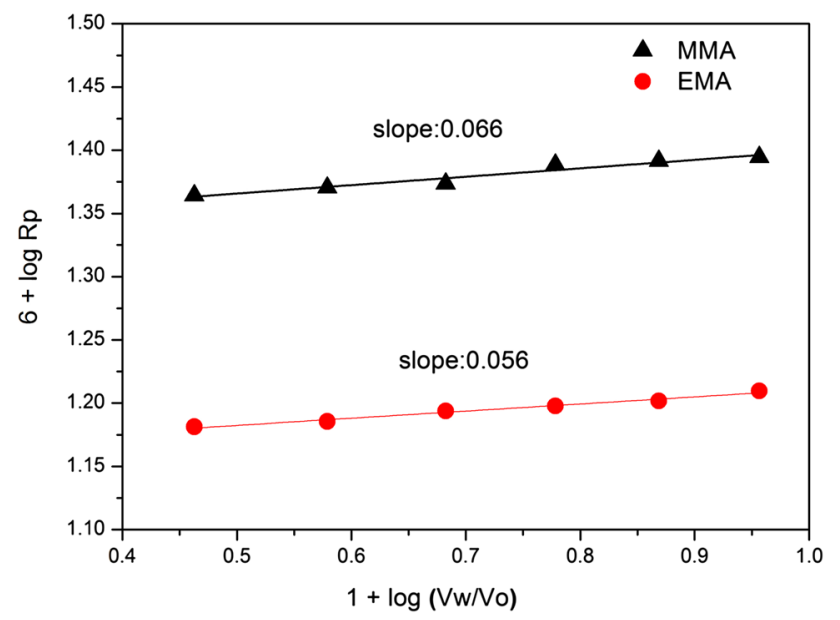

Fig. 8 Role of aqueous phase on the $R$ p. Reaction condition: [RMA]: $2.0 \mathrm{~mol} \mathrm{dm}^{-3}$; [PDS]: $2.0 \times 10^{-2} \mathrm{~mol} \mathrm{dm}^{-3}$; [TMBPEDBC]: $2.0 \times 10^{-2} \mathrm{~mol} \mathrm{dm}^{-3} ;\left[\mathrm{H}^{+}\right]: 0.50 \mathrm{~mol} \mathrm{dm}{ }^{-3} ;[\mu]: 0.20 \mathrm{~mol} \mathrm{dm}^{-3}$; Time: $50 \mathrm{~min}$; Temperature: $60 \pm 1{ }^{\circ} \mathrm{C}$

variation of aqueous phase was found to exert no significant change in the rate of polymerization for both alkyl methacrylates. It has been reported that the variation of aqueous phase on $R_{\mathrm{p}}$ was not affected significantly on phase-transfercatalyzed polymerization studies [18-27].

\section{Role of ionic strength and pH on $R_{p}$}

The role of ionic strength and $\mathrm{pH}$ is an important parameter in the polymerization reaction. The $\mathrm{pH}$ may influence the polymerization rate by coagulating the dispersed phase and affecting the initiation step. The adding and presented ions may cause a change in the ionic strength of the medium and it leads to a change in the initial and limiting conversion rate in polymerization. In this investigation, ionic strength and $\mathrm{pH}$ of the medium do not influence much on the polymerization rate. A similar kind of behavior was reported in phase-transfer-catalyzed polymerization of vinyl monomers [19-25].

\section{Relative reactivity of methyl and ethyl methacrylate on $R p$}

Generally, acrylate monomers can be polymerized either by free radical or ionic methods. The type of polymerization depends on the nature of reactive functional moiety present in monomer molecules. Most of the monomers will undergo polymerization with a radical initiator at varying rates. Methyl and ethyl methacrylate containing carbon-carbon double bond is responsible for polymerization and its carbonyl group is not prone to polymerization by radical initiators because of its polarized nature. However, the type of substitute on the $\mathrm{C}=\mathrm{C}$ double bond of acrylate monomers determines the reactivity and rate of polymerization. From the investigation, we observed that the rate of polymerization of EMA is greater compared to MMA (Table 3; Fig. 2). These trends can be explained in terms of inductive effect and steric effects. An increase in the rate from methyl to ethyl is attributed to the greater electron-donating tendency of ethyl $\left(-\mathrm{CH}_{2} \mathrm{CH}_{3}\right.$ of EMA) as compared with methyl group ( $-\mathrm{CH}_{3}$ group of MMA) [30]. An increased electron-donating tendency (of EMA) is facilitating the easy opening of the double bond and in this way increases the rate of addition of monomer to the polymer radical. The steric differences between MMA and EMA become insignificant in the rate of polymerization (steric effects may become important with more substituent) [31, 32]. The vinyl monomers such as MMA, EMA, n-BMA, styrene and acrylonitrile were polymerized using DPDS and $\mathrm{Cu}$ (II) system [33]. Among these monomers, EMA was polymerized easily compared to other monomers. The order of ease of

Table 3 Comparison of the relative reactivity of RMA

\begin{tabular}{l}
\hline Parameters \\
\hline Structure
\end{tabular}


polymerization is as follows: EMA $>$ MMA $>$ n-BMA $>$ styrene $>$ AN. This reported observation and the above explanation clearly support the polymerization reactivity of EMA and MMA under phase-transfer catalyst condition.

\section{Polymerization reaction mechanism}

On the basis of the observed experimental conditions, the polymerization reaction mechanism for alkylmethcrylates in the presence of multi-site PTC (TMBPEDBC) and potassium peroxydisulphate was proposed as shown in Scheme 4. Initially, the formation of $\mathrm{QS}_{2} \mathrm{O}_{8}$ took place in the aqueous phase on reaction between multi-site PTC $\left(\mathrm{QX}_{2}\right)$ and initiator $\left(\mathrm{K}_{2} \mathrm{~S}_{2} \mathrm{O}_{8}\right)$. The intermediate $\left(\mathrm{QS}_{2} \mathrm{O}_{8}\right)$ complex is decomposed and forms a radical anion at the interface whichinitiates the polymerization reaction.

The equilibrium constants $\left(K_{1}\right.$ and $\left.K_{2}\right)$ in the reactions in Eq. (1) to (3) and distribution constants ( $\alpha_{1}$ and $\alpha_{2}$ of QX and $\mathrm{QS}_{2} \mathrm{O}_{8}$ are defined as follows, respectively:

$$
\begin{aligned}
& K_{1}=\frac{\left[Q^{2+}\right]_{w}\left[X^{-}\right]_{w}^{2}}{[Q X]_{w}} \\
& K_{2}=\frac{\left[K^{+}\right]_{w}^{2}\left[S_{2} O_{8}^{2-}\right]_{w}}{\left[K_{2} S_{2} O_{8}\right]_{w}} \\
& K_{3}=\frac{\left[Q S_{2} O_{8}\right]_{w}}{\left[Q^{+}\right]_{w}\left[S_{2} O_{8}^{2-}\right]} \\
& \alpha_{1}=\frac{\left[Q^{2+} X_{2}^{-}\right]_{w}}{[Q X]_{o}}
\end{aligned}
$$

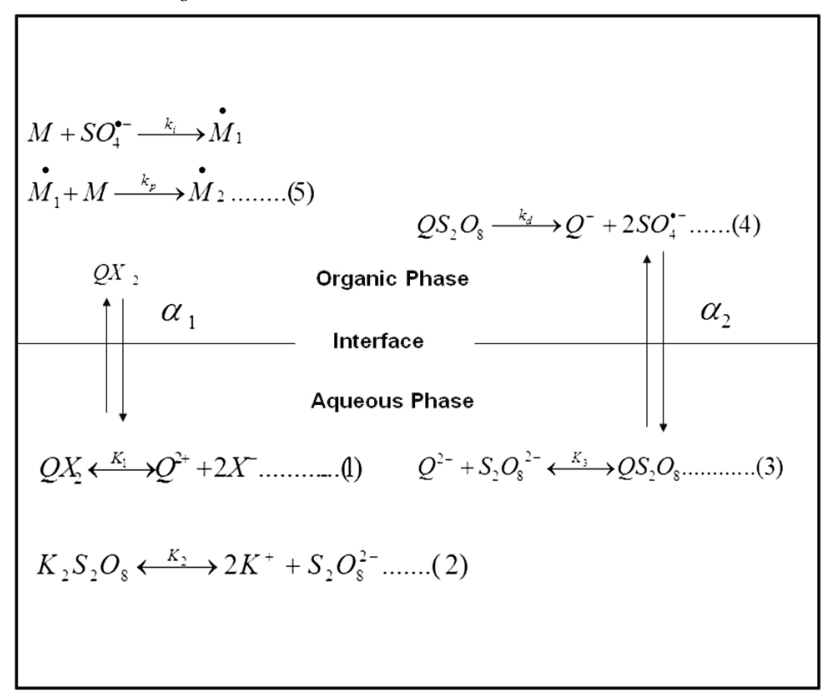

Scheme 4 Polymerization reaction mechanism of RMA-TMBPEDBC-PDS in two-phase system
$\alpha_{2}=\frac{\left[Q^{2+} S_{2} O_{8}^{2-}\right]_{w}}{\left[Q S_{2} O_{8}\right]_{o}}$

Applying general principles of free radical polymerization and steady-state hypothesis to radical species, the rate law for this mechanism can be derived $[22,28]$ as follows:

$R_{p}=k_{p}\left[\frac{k_{d} K_{3} f}{k_{t}}\right]^{1 / 2}\left[Q^{2+}\right]_{w}^{0.50}\left[S_{2} O_{8}^{2-}\right]_{w}^{0.60}[M]^{0.50}$

\section{Analysis of poly (alkyl methacrylates)}

\section{FT-IR analysis of poly (alkyl methacrylate)}

Figure 9 depicts the FT-IR spectrum of obtained PMMA and PEMA. The observed frequency confirms an ester functional stretching band of poly (methyl methacrylate) and poly (ethyl methacrylate) at 1732 and $1734 \mathrm{~cm}^{-1}$, respectively. The following bands were also observed in the spectra of PMMA $\left(\mathrm{cm}^{-1}\right)$ : 1128-1262 (C-O-C stretching band), 1458 (C-H deformation), 2952 (C-H stretching band) and PEMA $\left(\mathrm{cm}^{-1}\right)$ : $1260(\mathrm{C}-\mathrm{O}-\mathrm{C}$ stretching band $), 1470(\mathrm{C}-\mathrm{H}$ deformation), 3042 (C-H stretching band). All the characteristics bands corresponding to the synthesised poly (alkyl acrylates) were in good agreement with their pristine polymers [34-36].

\section{TG-DSC analysis of poly (alkyl methacrylate)}

TGA analysis is mainly used to examine thermal stability and degradation temperature of polymers. The thermal stabilities of poly (alkyl methacrylate) were studied by TG analysis. Figure 10 shows the TG curve of both polymers. The theromogram of PMMA and PEMA showed no weight

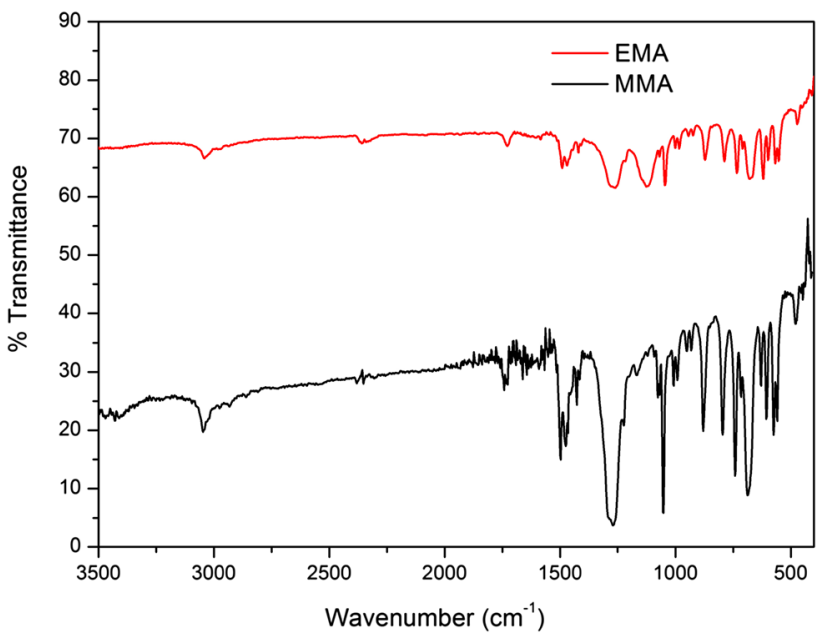

Fig. 9 FT-IR spectral analysis of poly (alkyl methacrylate) 
loss up to 150 and $200{ }^{\circ} \mathrm{C}$, respectively. PMMA showed a weight loss of about $17 \%$ in the range of $250-375{ }^{\circ} \mathrm{C}$ and in the third stage PMMA has a weight loss about $80.50 \%$ due to $375-420{ }^{\circ} \mathrm{C}$ and the remaining left as a residue. In PEMA thermogram, the first weight loss of the polymer is about $5.57 \%$ at $200-215^{\circ} \mathrm{C}$ is may bedue to absorbed water. In the second stage, the weight loss of $15.17 \%$ observed at $215-485^{\circ} \mathrm{C}$. The extensive degradation of polymer occurs at $485-576{ }^{\circ} \mathrm{C}$ with loss of $80 \%$ and then finally leaves residue of the polymer of the sample.

DSC analysis was carried out to observe the changes like fusion, glass transition temperature $\left(T_{\mathrm{g}}\right)$ and crystallization of polymers. The glass transition of amorphous polymers or amorphous domains of semi-crystalline polymers marks the change from a glassy to a rubbery state. The softening physical nature of the polymers was characterized by $T_{\mathrm{g}}$. Further, $T_{\mathrm{g}}$ depends on tacticity, molecular weight and solvent used. Figure 11 depicts the DSC thermogram curve of synthesised PMMA and PEMA. The glass transition temperature $\left(T_{\mathrm{g}}\right)$ was estimated at the inflection point between the onset and end set temperature. The glass transition temperature $\left(T_{\mathrm{g}}\right)$ value of PMMA and PEMA was observed at 107 and $65^{\circ} \mathrm{C}$, respectively [37].

\section{XRD analysis of poly (alkyl methacrylate)}

The XRD is generally used to delineate nature of polymers that is amorphous or crystalline in nature, etc. The d-spacing is determined from the peak position using Bragg's law: $n \lambda=2 \mathrm{~d} \sin \theta$, where $\lambda$ is the wavelength of the X-rays and $\mathrm{n}$ is positive integer. Figure 12 shows the XRD pattern of the synthesised PMMA and PEMA. The diffraction patterns of both polymers show very broad diffraction peak at low angle in the range of $10^{\circ}$ to $30^{\circ}$. A typical amorphous material exhibits few band of low intensities centred at $30^{\circ}$ and $42^{\circ}$ [38]. It revealed that both polymers have predominated amorphous nature.

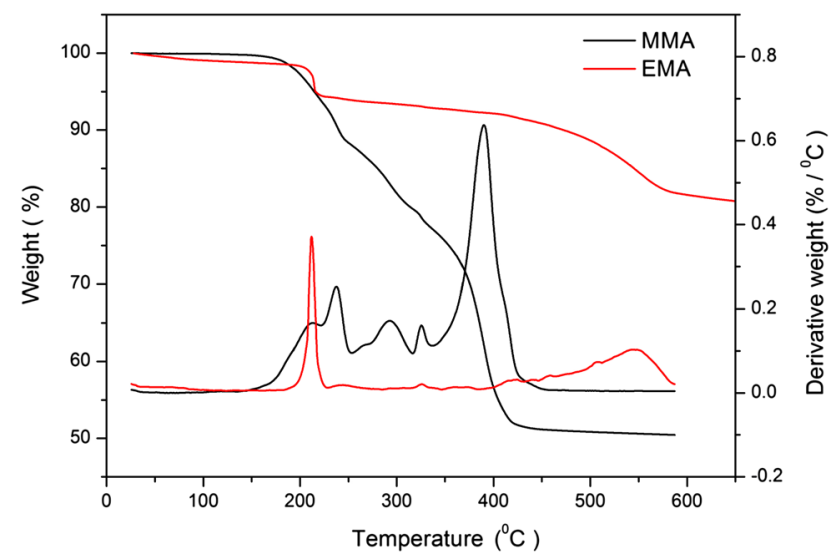

Fig. 10 Thermo gravimetric analysis of poly (alkyl methacrylate)

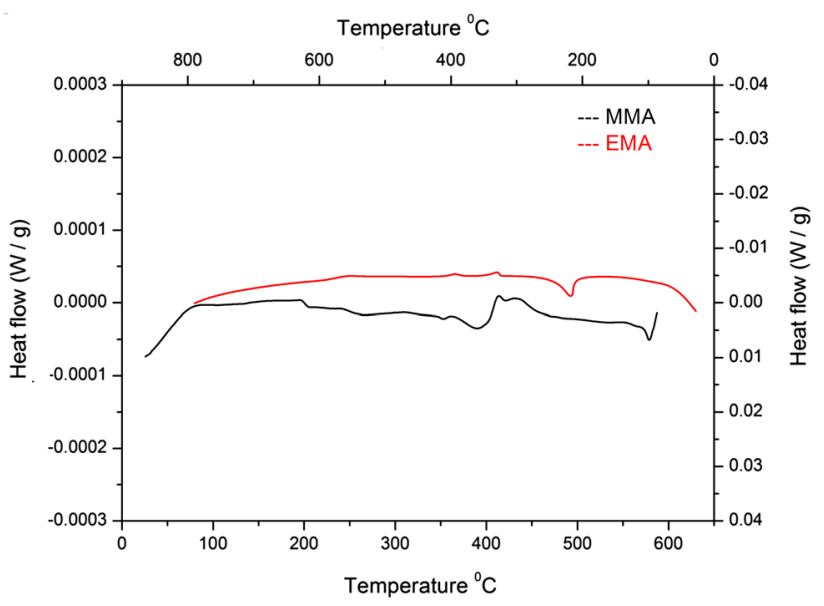

Fig. 11 DSC analysis of poly (alkyl methacrylate)

\section{Conclusions}

The new multi-site phase-transfer catalyst (TMBPEDBC) was synthesized and employed in polymerization of MMA and EMA using water-soluble initiator. The relative reactivity of MMA and EMA was explored under phase-transfer catalytic conditions by various reaction parameters. The results showed that the reactivity of EMA is faster than MMA. Increases in the rates from MMA to EMA are attributed to the greater electron-donating power of the ethyl group as compared with methyl. The steric difference between methyl and ethyl is probably unimportant. In addition to that, the reactivity of alkyl methacrylate was well supported by the activation energy of polymerization reaction. The obtained poly (alkyl methacrylate) was analyzed by thermal and spectral analyses. Herein the reported

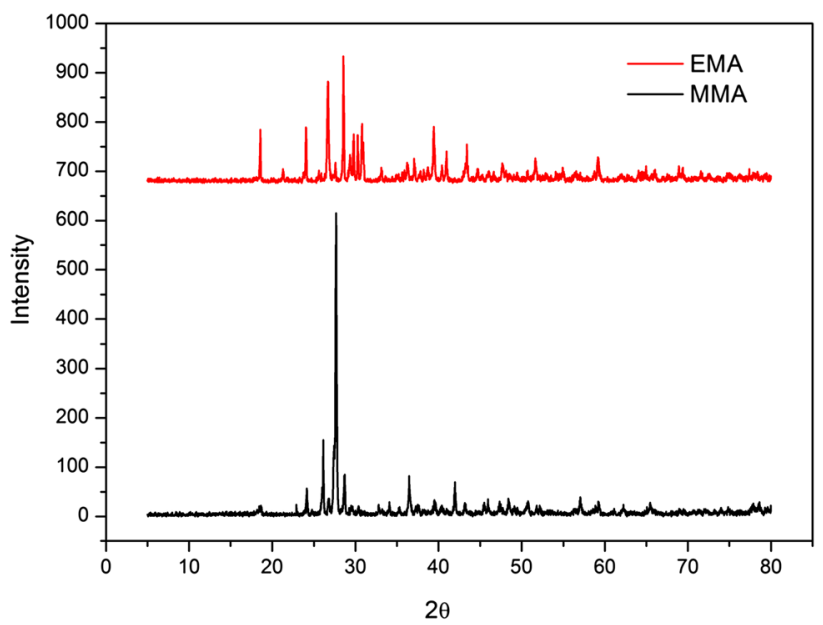

Fig. 12 XRD analysis of poly (alkyl methacrylate) 
polymerization process was followed half order with respect to monomer, initiator and catalyst. Phase-transfer catalyst showed great potential applications for synthesis of polymers and organic compounds. We believe that the integration of PTC with other technologies may pave a facile and promising avenue for obtaining high yield in heterogeneous system.

Acknowledgements The financial support from the Science and Engineering Research Board (DST-SERB) India under the young scientist start-up research grant (SB/FT/CS-008/2014) is gratefully acknowledged. Authors thank the Management of B S Abdur Rahman Crescent Institute of Science and Technology for providing facility.

\section{Compliance with ethical standards}

Conflict of interest The authors declare that there is no conflict of interest regarding the publication of this article.

Open Access This article is distributed under the terms of the Creative Commons Attribution 4.0 International License (http://creativeco mmons.org/licenses/by/4.0/), which permits unrestricted use, distribution, and reproduction in any medium, provided you give appropriate credit to the original author(s) and the source, provide a link to the Creative Commons license, and indicate if changes were made.

\section{References}

1. Park IJ, Lee SB, Choi CK (1994) Surface properties for poly (perfluoroalkylethyl methacrylate)/poly ( $n$-alkyl methacrylate)s mixtures. J Appl Polym Sci 54:1449-1454

2. McLain SJ, Sauer BB, Firment LE (1996) Surface properties and metathesis synthesis of block copolymers including perfluoroalkyl-ended polyethylenes. Macromolecules 29:8211-8219

3. Hawker CJ, Bosman AW, Harth Eva (2001) New polymer synthesis by nitroxide mediated living radical polymerizations. Chem Rev 101:3661-3668

4. Matyjaszewski K, Xia J (2001) Atom transfer radical polymerization. Chem Rev 101:2921-2990

5. Moad G, Rizzardo Ezio, Thang SH (2008) Toward living radical polymerization. Acc Chem Res 41:1133-1142

6. Ouchi M, Terashima T, Sawamoto M (2009) Transition metal-catalyzed living radical polymerization: toward perfection in catalysis and precision polymer synthesis. Chem Rev 109:4963-5050

7. Hawker CJ (1997) Living free radical polymerization: a unique technique for the preparation of controlled macromolecular architectures. Acc Chem Res 30:373-382

8. Moad G, Rizzardo Ezio, Thang SH (2008) Radical addition-fragmentation chemistry in polymer synthesis. Polymer 49:1079-1131

9. Lowe AB, McCormick CL (2007) Reversible addition-fragmentation chain transfer (RAFT) radical polymerization and the synthesis of water-soluble (co)polymers under homogeneous conditions in organic and aqueous media. Prog Polym Sci 32:283-351

10. Rosen BM, Percec V (2009) Single-electron transfer and singleelectron transfer degenerative chain transfer living radical polymerization. Chem Rev 109(11):5069-5119

11. Yamago Shigeru (2009) Precision polymer synthesis by degenerative transfer controlled/living radical polymerization using organotellurium, organostibine, and organobismuthine chaintransfer agents. Chem Rev 109(11):5051-5068
12. Dariusz B, Mateusz G, Grzegorz B, Jacek W, Stanislaw W (2010) Preparation of polymers under phase transfer catalytic conditions. Org Proc Res Dev 14:669-683

13. Starks CM, Liotta C, Halpern M (1994) Phase transfer catalysis: fundamentals. applications and perspectives. Chapman and Hall, New York

14. Weber WP, Gokel GW (1977) Phase transfer catalysis in organic synthesis. Springer, New York

15. Makosza M, Fedorynski M (2003) Phase transfer catalysis. Catal Rev 45:321-367

16. Balakrishnan T, Damodarkumar S (2000) Phase transfer catalysis: free radical polymerization of acrylonitrile using peroxymonosulphate/tetrabutylphosphonium chloride catalyst system: a kinetic study. J Appl Polym Sci 76:1564-1571

17. Thajudeen H, Jamal Ahamed VS, Shabeer TK (2008) Phase transfer catalysis: polymerization kinetics and mechanism of methyl acrylate. Int J Polym Mater 57:904-917

18. Damodarkumar S, Perumal A (2007) Kinetics of free radical polymerization of methylacrylate initiated by potassium peroxomonosulphate and tetrabutyl phosphonium chloride. Asian J Chem 19:5081-5088

19. Murugesan V, Umapathy MJ (2016) Phase transfer catalyst aided radical polymerization of n-butyl acrylate in two phase system-a kinetic study. Int J Ind Chem 7:441-448

20. Murugesan V, Marimuthu E, Yoganand KS, Umapathy MJ (2017) Multi-site phase transfer catalysed radical polymerization of methyl methacrylate in mixed aqueous-organic medium-a kinetic study. Int J Ind Chem 8:241-251

21. Vajjiravel M, Umapathy MJ, Bharathbabu M (2007) Polymerization of acrylonitrile using potassium peroxydisulfate as an initiator in the presence of a multisite phase-transfer catalyst: a kinetic study. J Appl Polym Sci 105:3634-3639

22. Vajjiravel M, Umapathy MJ (2008) Synthesis and characterization of multi-site phase transfer catalyst: application in radical polymerisation of acrylonitrile-a kinetic study. J Polym Res 15:27-36

23. Vajjiravel M, Umapathy MJ (2008) Free radical polymerisation of methyl methacrylate initiated by multi-site phase transfer catalyst-a kinetic study. Colloid Polym Sci 286:729-738

24. Vajjiravel M, Umapathy MJ (2010) Synthesis, characterization and application of a multi-site phase transfer catalyst in radical polymerization of n-butyl methacrylate-a kinetic study. Int $\mathbf{J}$ Polymeric Mater 59:647-662

25. Vajjiravel M, Umapathy MJ (2011) Kinetics of radical polymerization of glycidyl methacrylate initiated by multi-site phase transfer catalyst-potassium peroxydisulfate in two-phase system. J Appl Polym Sci 120:1794-1799

26. Vajjiravel M, Umapathy MJ (2009) Kinetics and mechanism of multi-site phase transfer catalyzed radical polymerization of ethyl methacrylate. Int J Polym Mater 58:61-76

27. Vajjiravel M, Umapathy MJ (2010) Multi-site phase transfer catalyzed radical polymerization of n-butyl methacrylate: a kinetic study. Chem Eng Commun 197:352-365

28. Marimuthu E, Murugesan V (2017) Influence of ultrasonic condition on phase transfer catalyzed polymerization of methyl methacrylate in two phase system-a kinetic study. Ultrason Sonochem 38:560-569

29. Marimuthu E, Murugesan V (2017) Ultrasonic condition boost up the rate of phase transfer catalyzed polymerization of acrylonitrile in two phase system. Appl Petrochem Res 7:85-96

30. McCurdy KG, Laidler KJ (1964) Rates of polymerization of acrylates and methacrylates in emulsion systems. Can J Chem 42:825-829

31. Otsu T, Ito T, Imato M (1967) Reactivities of alkylmethacrylates in their radical polymerization. J Polym Sci Part C 16:2121-2130 
32. Otsu T, Ito T, Imato M (1965) Reactivities of alkylmethacrylates in their radical polymerization. Polym Lett 3:113-117

33. Ouchi T, Beika N, Imoto M (1982) Vinyl polymerization CDVII polymerization of methyl methacrylate initiated by diphenyldisulphide and $\mathrm{Cu}$ (II) ion. Polymer J 14:155-160

34. Sperling LH (2006) Introduction to physical polymer science. Wiley, New York

35. Kroschwitz JI (1990) Polymer: polymer characterization and analysis. Wiley, New York

36. Schrader B (1995) Infrared and Raman spectroscopy: methods and applications. VCH, Germany

37. Roth CB, Pound A, Kamp SW, Murray CA, Dutcher JR (2006) Molecular-weight dependence of the glass transition temperature of freely-standing poly (methyl methacrylate) films. Eur Phys J E 20:441-448

38. Saladino ML, Motaung TE, Luyt AS, Spinella A, Nasillo G, Caponetti E (2012) The effect of silica nanoparticles on the morphology, mechanical properties and thermal degradation kinetics of PMMA. Polym Degrad Stab 97:452-459

Publisher's Note Springer Nature remains neutral with regard to jurisdictional claims in published maps and institutional affiliations. 